\title{
Dye Incorporation in Polyphosphate Gels: Synthesis and Theoretical Calculations
}

\author{
Jordan Del Nero ${ }^{\mathrm{a} *}$, André Galembeck ${ }^{\mathrm{b}}$, Sidicleia Bezerra Costa Silva ${ }^{\mathbf{b}}$, \\ José Augusto Pereira da Siva ${ }^{b}$ \\ ${ }^{a}$ Departamento de Física, Universidade Federal do Pará (UFPA), \\ 66075-110 Belém - PA, Brazil \\ ${ }^{\mathrm{b}}$ Departamento de Química Fundamental, Universidade Federal de Pernambuco (UFPE), \\ 50670-901 Recife - PE, Brazil
}

Received: October 10, 2002; Revised: February 23, 2003

\begin{abstract}
In this work we described theoretical calculations on the electronic structure and optical properties of the dyes crystal violet and malachite green based in semiempirical methods (Parametric Method 3 and Intermediate Neglect of Differential Overlap / Spectroscopic - Configuration Interaction) and the synthesis of a new hybrid material based upon the incorporation of these dyes in an aluminum polyphosphate gel network. The samples are nearly transparent, free-standing thick films. The optical properties of the entrapped dyes are sensitive to chemical changes within the matrix caused either by gel aging or external stimulli such as exposition to acidic and basic vapors that can percolate within the matrix. Our theoretical modeling is in good agreement with the experimental results for the dyes.
\end{abstract}

Keywords: sol-gel, electronic structure, theoretical and experimental absorption spectra, semiempirical/CI calculations, malachite green, crystal violet

\section{Introduction}

The sol-gel process provides interesting paths to synthesize organic-inorganic hybrid materials, in which one can take advantage of the properties of each component individually and, also, reach synergistic properties between them. The organic and inorganic components may either exchange weak interactions like hydrogen bonding, van de Waals forces and electrostatic forces or be linked through chemical bonds. The inorganic to organic relative amount may change significantly ${ }^{1}$.

Silica-based networks are the most widely used inorganic fraction because the precursors are commercially available and/or can be easily modified. Their chemical reactivity is well understood and the synthesis may be carried out to form transparent films, fibers or monoliths with good mechanical properties ${ }^{2}$.

Polyphosphate-based materials are also attractive as hosts for the design of inorganic hybrid composites as they can be synthesized as particles containing closed pores ${ }^{3}$, nanoparticle ${ }^{4}$, transparent gels $^{5}$, glasses ${ }^{6}$ and hybrid net-

*e-mail: jordan@ufpa.br

Trabalho apresentado no $1^{\circ}$ Congresso da Sociedade Brasileira em Materiais, Rio de Janeiro, Julho de 2002. works ${ }^{7}$. These materials may present a broad range of chemical compositions, with many different morphologies which allow us to envisage the entrapment of compounds in mild reaction conditions, thus preventing degradation of the active species.

Polyphosphate gels can be synthesized as transparent, flexible free-standing films. The gelation derives from the association of the polyphosphate polyanions with metallic cations, leading to a supramolecular ionic swollen network. In fact, these cations $(\mathrm{Ca}, \mathrm{Zr}, \mathrm{Al}, \mathrm{Fe})$ act as bridges between different polyphosphate chains and, hence, the gel cohesion is a consequence of electrostatic interactions rather than the covalent bonding which develops as a result of the polycondensation reactions that proceed to give silica-based networks, starting from alkoxide precursors.

We have reported, recently, the synthesis of new hybrid materials in which a polyphosphate gel network was used as the host structure for the incorporation of rare-earth cryptates $^{8}$, polyaniline ${ }^{9}$ and the dyes methyl orange ${ }^{10}$ and malachite green ${ }^{11}$, in order to develop new optical materials. 
Crystal violet (CV) and malachite green (MG) are triaryl-methane dyes with similar chemical structures (Fig. 1). They are used as acidic-base indicators, stain compounds in histology and as flourophores in immunoassays.

$\mathrm{CV}$ tends to dimerize in aqueous solution depending upon the dye concentration. The monomer and dimer give different absorption bands in the visible spectrum. Also, two kinds of molecular orientation were described for CV dimerization in the literature: (i) the molecules are parallel, and; (ii) there is a superposition of the nitrogen atom from one dymethyl-amine group to the aromatic ring (anti-parallel). $\mathrm{MG}$ structure is very similar to $\mathrm{CV}$, but no dimerization is reported in the literature ${ }^{12,13}$.

Alberti et al. reported the intercalation of crystal violet both in the external surface and in the interlayer space of azirconium phosphate $(\alpha-\mathrm{ZrP})$. They observed both dimerization and protonation by the acidic $\mathrm{P}-\mathrm{OH}$ groups within the dye host structure. Also, the design of optical sensors for ammonia and humidity detection were performed by dispersing this dye on Nafion ${ }^{\circledR}$ films ${ }^{14}$. Another chemical optical sensor for ammonia in which a sulfonephthalein (also a substituted triaryl-methane) dye was immobilized in sol-gel derived silica thin films was also reported. In the later, a glassy film of the organically modified silicate was deposited in optical fibers ${ }^{15}$.

In this work we investigated theoretically the $\mathrm{CV}, \mathrm{MG}$ and CV-MG dimerization in order to optimize the dimer geometry and understanding its spectroscopic properties. We also synthesized new hybrid materials by the incorporation of these dyes within polyphosphate gels in order to get new optical sensors.

\section{Methodology}

\subsection{CV-MG/Polyphosphate Hybrids Synthesis}

Crystal violet + malachite green/aluminum polyphosphate (CV-MG/APP) composites were synthesized starting from sodium polyphosphate $(2.0 \mathrm{~mol} / \mathrm{l}$, in $\mathrm{P})$ and aluminum nitrate $(1.0 \mathrm{~mol} / \mathrm{l})$ aqueous solutions. The $\mathrm{MG}$ to $\mathrm{CV}$ ratio in the dye aqueous solution was $[\mathrm{MG}] /[\mathrm{CV}]=5.0$, which was adjusted in order to give similar absorbances from the two dye solutions. The total CV and MG concentration were, respectively, $2.0 \times 10^{-5}$ and $1.0 \times 10^{-4} \mathrm{~mol} / \mathrm{l}$. The average polyphosphate chain length was 10.6 , determined by ${ }^{31} \mathrm{P}$ NMR.

In a typical synthesis, the MG-CV $(1 \mathrm{ml})$ solution was previously added to $10 \mathrm{ml}$ of the polyphosphate solution, followed by the addition $10 \mathrm{ml}$ of the $\mathrm{Al}^{3+}$ solution under strong stirring. In some cases, sodium hydroxide solution was also added to raise the $\mathrm{pH}$. The phosphorus to aluminum ratio $(\mathrm{P} / \mathrm{Al})$ ranged from 2.0 to 2.8 .

Gelation occurs under ambient atmosphere at room temperature within $1 \mathrm{~min}$. The gel was stirred for $30 \mathrm{~min}$ and centrifuged for $1 \mathrm{~h}$ at $10,000 \times \mathrm{g}$. Transparent greenishblue samples form after 1 to 3 days. Free-standing films were prepared spreading the gel samples on a flat surface and letting them dry at room temperature in a moisturecontrolled environment ( $50 \%$ relative humidity) for one week.

The samples were exposed to $\mathrm{HCl}$ and $\mathrm{NH}_{3}$ vapors, from $6 \mathrm{~mol} / \mathrm{l}$ solutions heated at $60{ }^{\circ} \mathrm{C}$, in a closed environment order to investigate their sensitivity to acidic and basic vapors.

The MG-CV/PP free-standing thick film samples were characterized by absorption spectra using a using an UV-visible spectrophotometer Perkin-Elmer LAMBDA 6 model 2688-002, within the 200-800 $\mathrm{nm}$ range.

\subsection{Theoretical Calculations}

Semiempirical calculations at the PM $3^{16}$ levels were performed for the guest molecules $\mathrm{CV}$ and $\mathrm{MG}$ and the corresponding fully optimized geometry could be determined. Standard MOPAC ${ }^{17}$ parameters were used, except for the convergence criterion, for which a maximum step size of 0.005 was adopted. For these molecules, a planar configuration was assumed as the starting point for the geometry optimization calculations. A RHF criterion was adopted because the compounds investigated have a positive ion in the second phenyl ring, which corresponds to a closed shell system.

Absorption spectrum calculations of the structures presented in Fig. 1 were performed using the ZINDO package $^{18-21}$ with geometries fully optimized at the PM3 level and the absorption spectra were calculated at the Intermediate Neglect of Differential Overlap/Spectroscopic - Configuration Interaction (INDO/S-CI) level, and the parameters chosen to give the best description of the UV-visibleNIR optical transitions for those molecules ${ }^{22-25}$. In this case, the level of approximation was utilized within a restricted closed-shell Hartree-Fock approach to determine the ground state. Approximately 280 configurations were investigated for each molecule, including singlet and doublet states.
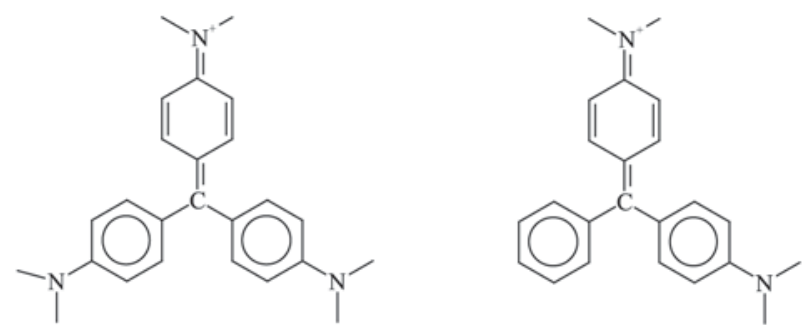

Figure 1. (a) Crystal violet (CV); (b) malachite green (MG) chemical structures. 
For molecule dimerization, we started from the geometries optimized with semiempirical calculations at the PM3 level and separated by 2.6 angstroms according with our previous calculations ${ }^{11}$.

\section{Results And Discussions}

Figure 2 presents the absorption spectra of $\mathrm{CV}, \mathrm{MG}$ and the MG-CV aqueous solutions. The spectrum of the CV solution shows two maxima near 544 and $584 \mathrm{~nm}$. The first one is attributed to $\mathrm{CV}$ dimerization ${ }^{26}$. The spectrum of the MG-CV mixture is the sum of the individual solutions.

After the centrifugation of the samples a supernatant solution is expelled from the solid gel, which is called synerisis liquid. A spectrum is also presented in Fig. 2e. As gelation proceeds in acidic medium (0.5 to 2.0), the supernatant solution spectrum is compared to $\mathrm{MG}$ and $\mathrm{CV}$ solutions spectra in the same conditions, which are very similar (Fig. 2c and 2d). This was done in order to investigate if one of the dyes is preferentially incorporated to the gel matrix, which is not the case as the supernatant spectrum showed contributions from both dyes.

The spectrum of the MG-CV/PP hybrid is presented in Fig. 3. The spectrum is similar to the MG-CV solution, which is greenish-blue. Both dyes were incorporated to the gel matrix. When these samples were exposed to $\mathrm{HCl}$ vapors, the films turned to green as presented in Fig. 3b. This sample presented two absorptions: (i) the first one has two maxima at 416 and $438 \mathrm{~nm}$, ascribed to $\mathrm{CV}$ and MG in acidic media; (ii) $640 \mathrm{~nm}$, due to $\mathrm{CV}$ protonation to give $\mathrm{CV}-\mathrm{H}$. The absorption at $640 \mathrm{~nm}$ has an asymmetry, which

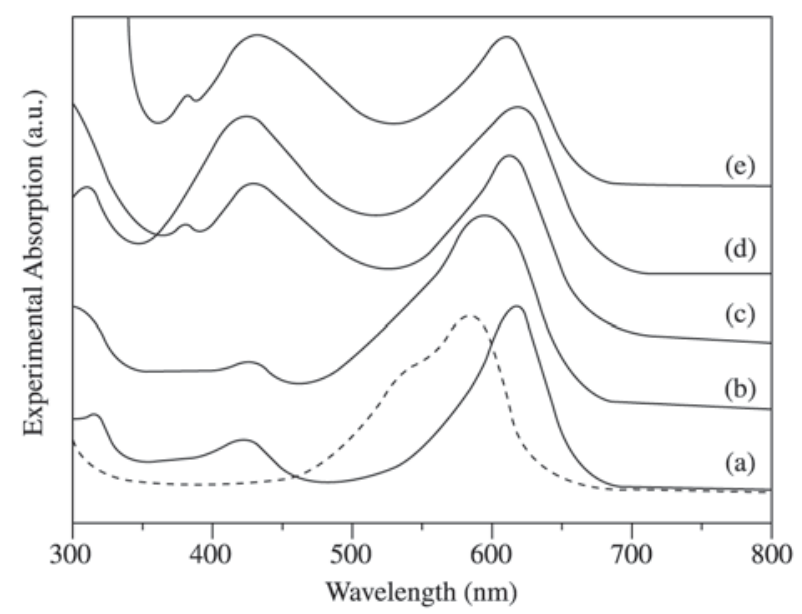

Figure 2. Absorption spectra. (a) MG (solid line) and CV (dashed line) aqueous solutions; (b) MG-CV aqueous solution; (c) MG in acidic medium; (d) CV in acidic medium; (e) supernatant liquid expelled from the gel after centrifugation (synerisis liquid). may result also from $\mathrm{MG}$ in acidic medium ${ }^{26}$. Hence, both dyes can be protonated by sample exposition to acidic vapors. Exposition to basic ammonia vapors leads to deprotonation of those dyes and the original color is restored.

MG-CV/PP samples presented the same color change if dried at 50\% humidity (green samples). The spectrum of a dried MG-CV gels is presented in Fig. 3c. The maximum is centered at $630 \mathrm{~nm}$ and the band is broader than in the former case, in which the sample was exposed to acidic vapors. The original greenish-blue color is restored if the samples were exposed to moisture-rich (> 80\%) environment.

The gel tends absorb and desorb water in reversible way. Fresh samples presents higher water content than samples dried under 50\% relative humidity. As the sample dries, the water amount within the gel liquid phase decreases, which should increase the acidity of this phase. The color will develop if the $\mathrm{pH}$ change related to this process is enough to protonate/deprotonate the dye which is incorporated in the gel matrix.

$\mathrm{CV}$ presents a violet-blue to green color change when the $\mathrm{pH}$ decreases from 2.0 to 1.0. MG turns to yellow if the $\mathrm{pH}$ is lower than 1.0. This means that the dyes acted as internal sensors for the aqueous phase of the gel, which is within the 1.0 to 2.0 range.

Once CV and MG are cationic species, they should have affinity with polyphosphate polyanion chains. This interaction leads to a phenomenon called metachromasy in which the absorption bands of the dye is dislocated in relation to aqueous solution. In aqueous solution, the maximum absorption for $\mathrm{CV}$ is located at $590 \mathrm{~nm}^{11}$. A shift to $603 \mathrm{~nm}$ is

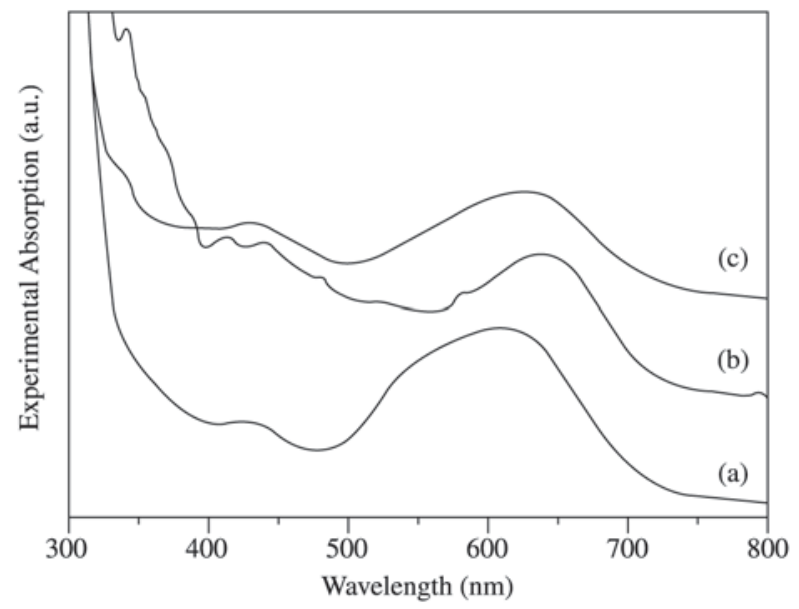

Figure 3. Absorption spectra. (a) MG-CV/APP gel (as-prepared); (b) sample (a) after exposition to $\mathrm{HCl}$ vapors for $30 \mathrm{~min}$; (c) sample (a) dried at $47 \%$ relative humidity for $48 \mathrm{~h}$. 
observed (not shown) if $\mathrm{CV}$ is added to a $2.0 \mathrm{~mol} / \mathrm{l}$ polyphosphate solution, used to synthesize the gel,. This means that the guest molecule interacts with these chains even before its incorporation within the gel. After dye incorporation in the maximum shifts further to 611 (Fig. 3).

The interactions between host and guest molecules are electrostatic and contribute to stabilize the hybrid, but the optical properties of the guest molecule is not significantly altered upon its incorporation within the matrix, which is desirable if one is interested in a solid state chemical sensor based on $\mathrm{CV}$ and MG dyes.

Figure 4 presents the main transitions contributing to the optical spectra of dye molecules. The corresponding theoretical absorption spectra were obtained by broadening each of these principal transitions by a suitably weighted Gaussian function normalized to the calculated oscillator strengths ${ }^{27}$.

For both, $\mathrm{CV}+$ and $\mathrm{MG}+$, the first and second optical transitions are essentially a HOMO to LUMO transition $(\mid \mathrm{H} \rightarrow \mathrm{L}>)$ and HOMO-1 to LUMO transition $(\mid \mathrm{H}-1 \rightarrow \mathrm{L}>)$, respectively. Those transitions appears at different regions of the spectra, the first one for $\mathrm{CV}+[\mathrm{MG}+]$ at 427.3 [448.2] $\mathrm{nm}$ with oscillator strength (o.s.) equal to 0.88 [1.06] and the second one for $\mathrm{CV}+[\mathrm{MG}+]$ at 424.8 [360.7] and o.s. equal to 0.89 [0.48]. For this reason the band at this region of spectrum is more pronounced for $\mathrm{CV}+$ molecule and it appears an extra band for MG+ molecule (360 $\mathrm{nm}$ region). Details for the three additional bands, it is presented in Table 1.

Figure 5 shows the theoretical absorption spectra for parallel dimer of (a) $\mathrm{CV}+/ \mathrm{CV}+$, (b) $\mathrm{MG}+/ \mathrm{MG}+$ and

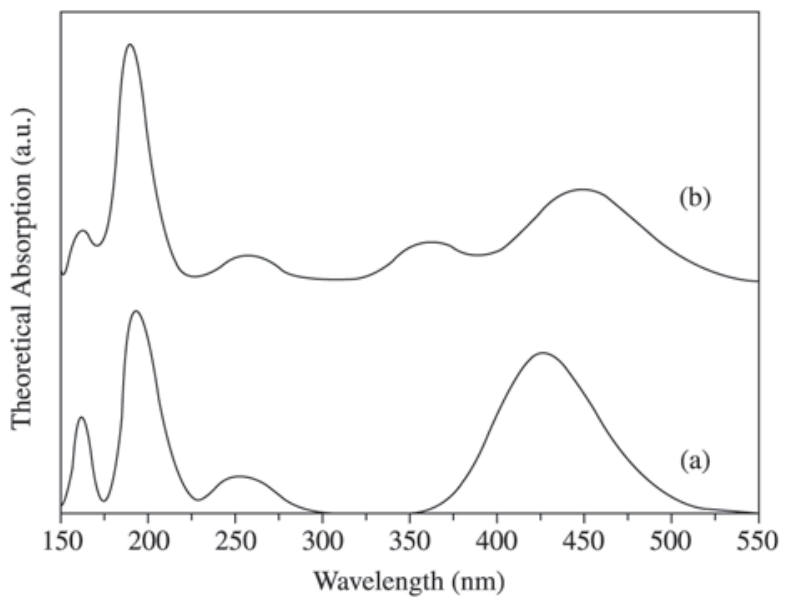

Figure 4. Theoretical absorption spectra. (a) $\mathrm{CV}^{+}$and (b) $\mathrm{MG}^{+}$ molecules by INDO/S-CI using the semiempirical method calculated for optimized geometries.
Table 1. Main electronic transitions as determined from INDO/S-CI calculations based on optimized ground-state geometry by semiempirical method. (nm, o.s., // and anti-// mean the wavelength of transition, the oscillator strength, the parallel and anti-parallel interaction between the molecules, respectively.)

\begin{tabular}{|c|c|c|c|}
\hline Molecule & $\mathrm{nm}$ & o.s. & Absorption \\
\hline $\mathrm{CV}^{+}$ & $\begin{array}{l}427.3 \\
424.8 \\
196.2 \\
188.7 \\
188.2 \\
\end{array}$ & $\begin{array}{l}0.8803 \\
0.8911 \\
0.3498 \\
0.6493 \\
0.5674 \\
\end{array}$ & $\begin{array}{c}0.68 \mid \mathrm{H} \rightarrow \mathrm{L}> \\
-0.68 \mid \mathrm{H}-1 \rightarrow \mathrm{L}> \\
0.33 \mid \mathrm{H}-2 \rightarrow \mathrm{L}+2> \\
-0.48 \mid \mathrm{H}-3 \rightarrow \mathrm{L}+3> \\
-0.47 \mid \mathrm{H}-4 \rightarrow \mathrm{L}+3>\end{array}$ \\
\hline$\overline{\mathrm{MG}^{+}}$ & $\begin{array}{l}448.2 \\
360.7 \\
197.1 \\
189.2 \\
188.3 \\
\end{array}$ & $\begin{array}{l}1.0681 \\
0.4816 \\
0.3452 \\
0.7573 \\
0.5606\end{array}$ & $\begin{array}{c}0.68 \mid \mathrm{H} \rightarrow \mathrm{L}> \\
-0.63 \mid \mathrm{H}-1 \rightarrow \mathrm{L}> \\
0.36 \mid \mathrm{H}-5 \rightarrow \mathrm{L}+1> \\
-0.35 \mid \mathrm{H}-3 \rightarrow \mathrm{L}+2> \\
0.35 \mid \mathrm{H}-2 \rightarrow \mathrm{L}+2>\end{array}$ \\
\hline$/ / \mathrm{CV}^{+} / \mathrm{CV}^{+}$ & $\begin{array}{l}483.6 \\
449.0 \\
444.8 \\
424.7 \\
402.1 \\
\\
386.0\end{array}$ & $\begin{array}{l}0.0574 \\
0.0954 \\
0.2217 \\
0.7681 \\
1.6242 \\
0.6955\end{array}$ & $\begin{array}{c}0.48 \mid \mathrm{H} \rightarrow \mathrm{L}> \\
-0.33 \mid \mathrm{H}-1 \rightarrow \mathrm{L}> \\
0.44 \mid \mathrm{H}-2 \rightarrow \mathrm{L}> \\
-0.53 \mid \mathrm{H} \rightarrow \mathrm{L}+1> \\
0.37 \mid \mathrm{H} \rightarrow \mathrm{L}> \\
-0.40 \mid \mathrm{H}-1 \rightarrow \mathrm{L}+1> \\
0.34 \mid \mathrm{H}-2 \rightarrow \mathrm{L}+1> \\
0.35 \mid \mathrm{H}-3 \rightarrow \mathrm{L}+1> \\
-0.50 \mid \mathrm{H}-4 \rightarrow \mathrm{L}>\end{array}$ \\
\hline$\overline{\mathrm{MG}^{+} / \mathrm{MG}^{+}}$ & $\begin{array}{l}557.9 \\
411.8 \\
\\
232.0 \\
229.0 \\
175.7 \\
\end{array}$ & $\begin{array}{l}0.1216 \\
2.1972 \\
0.2656 \\
0.8498 \\
0.4272\end{array}$ & $\begin{array}{c}-0.67 \mid \mathrm{H} \rightarrow \mathrm{L}> \\
0.46 \mid \mathrm{H}-1 \rightarrow \mathrm{L}+1> \\
0.49 \mid \mathrm{H}-2 \rightarrow \mathrm{L}> \\
0.48 \mid \mathrm{H}-1 \rightarrow \mathrm{L}+2> \\
-0.34 \mid \mathrm{H}-3 \rightarrow \mathrm{L}+2> \\
0.32 \mid \mathrm{H}-9 \rightarrow \mathrm{L}+4>\end{array}$ \\
\hline$\overline{\mathrm{CV}^{+} / \mathrm{MG}^{+}}$ & $\begin{array}{l}453.0 \\
422.2 \\
407.3 \\
\\
358.4 \\
243.6 \\
\end{array}$ & $\begin{array}{l}0.1512 \\
0.6894 \\
1.9387 \\
0.8088 \\
0.2277\end{array}$ & $\begin{array}{c}0.56 \mid \mathrm{H} \rightarrow \mathrm{L}> \\
0.54 \mid \mathrm{H}-1 \rightarrow \mathrm{L}+1> \\
-0.33 \mid \mathrm{H}-2 \rightarrow \mathrm{L}+1> \\
0.33 \mid \mathrm{H} \rightarrow \mathrm{L}> \\
-0.53 \mid \mathrm{H}-2 \rightarrow \mathrm{L}+1> \\
0.62 \mid \mathrm{H}-3 \rightarrow \mathrm{L}> \\
-0.33 \mid \mathrm{H}-1 \rightarrow \mathrm{L}+6> \\
0.33 \mid \mathrm{H}-2 \rightarrow \mathrm{L}+3>\end{array}$ \\
\hline$\overline{\text { Anti } / / \mathrm{CV}^{+} / \mathrm{CV}^{+}}$ & $\begin{array}{l}430.3 \\
427.2 \\
406.6 \\
\\
254.0 \\
246.6 \\
186.5 \\
\end{array}$ & $\begin{array}{l}0.6808 \\
1.3674 \\
1.8533 \\
\\
0.3794 \\
0.4485 \\
0.4872\end{array}$ & $\begin{array}{c}0.35 \mid \mathrm{H} \rightarrow \mathrm{L}+1> \\
-0.59 \mid \mathrm{H}-1 \rightarrow \mathrm{L}> \\
-0.58 \mid \mathrm{H} \rightarrow \mathrm{L}+1> \\
-0.36 \mid \mathrm{H}-1 \rightarrow \mathrm{L}> \\
0.49 \mid \mathrm{H}-2 \rightarrow \mathrm{L}+1> \\
0.46 \mid \mathrm{H}-3 \rightarrow \mathrm{L}> \\
-0.36 \mid \mathrm{H}-2 \rightarrow \mathrm{L}+4> \\
0.33 \mid \mathrm{H} \rightarrow \mathrm{L}+8> \\
-0.33 \mid \mathrm{H}-4 \rightarrow \mathrm{L}+8>\end{array}$ \\
\hline $\mathrm{MG}+/ \mathrm{MG}+$ & $\begin{array}{l}452.9 \\
366.2 \\
257.5\end{array}$ & $\begin{array}{l}0.8883 \\
0.5601\end{array}$ & $\begin{array}{c}0.51 \mid \mathrm{H} \rightarrow \mathrm{L}> \\
0.44 \mid \mathrm{H}-1 \rightarrow \mathrm{L}+1> \\
0.44 \mid \mathrm{H}-2 \rightarrow \mathrm{L}> \\
-0.41 \mid \mathrm{H}-3 \rightarrow \mathrm{L}+1> \\
-0.42 \mid \mathrm{H} \rightarrow \mathrm{L}+4>\end{array}$ \\
\hline$\overline{\mathrm{CV}+/ \mathrm{MG}+}$ & $\begin{array}{l}430.7 \\
425.7 \\
398.7 \\
\\
359.0 \\
251.1 \\
186.9\end{array}$ & $\begin{array}{l}1.1998 \\
1.2637 \\
0.7633 \\
\\
0.4287 \\
0.4657 \\
0.4773\end{array}$ & $\begin{array}{c}-0.64 \mid \mathrm{H} \rightarrow \mathrm{L}+1> \\
0.55 \mid \mathrm{H}-1 \rightarrow \mathrm{L}> \\
-0.38 \mid \mathrm{H}-1 \rightarrow \mathrm{L}+1> \\
0.55 \mid \mathrm{H}-2 \rightarrow \mathrm{L}+1> \\
-0.62 \mid \mathrm{H}-3 \rightarrow \mathrm{L}> \\
-0.39 \mid \mathrm{H}-1 \rightarrow \mathrm{L}+3> \\
0.34 \mid \mathrm{H}-4 \rightarrow \mathrm{L}+7>\end{array}$ \\
\hline
\end{tabular}


(c) $\mathrm{CV}+/ \mathrm{MG}+$ molecules interacting. The way of interaction for these molecules is following the steps discussed by theoretical methodology. For $\mathrm{CV}+/ \mathrm{CV}+$ (Fig. 5a) molecules interacting we can see the strongest band at $\approx 400 \mathrm{~nm}$. The most important transitions for this interaction correspond to a mixing of the transitions $|\mathrm{H} \rightarrow \mathrm{L}>,| \mathrm{H}-1 \rightarrow \mathrm{L}>, \mid \mathrm{H}-2 \rightarrow \mathrm{L}>$, $\mid \mathrm{H} \rightarrow \mathrm{L}+1>$ and $\mid \mathrm{H}-1 \rightarrow \mathrm{L}+1>$. Note that, those transitions came from the interaction between the two $\mathrm{CV}+$ molecules because for a single molecule the first transition is composed by only a $\mid \mathrm{H} \rightarrow \mathrm{L}>$ transition.

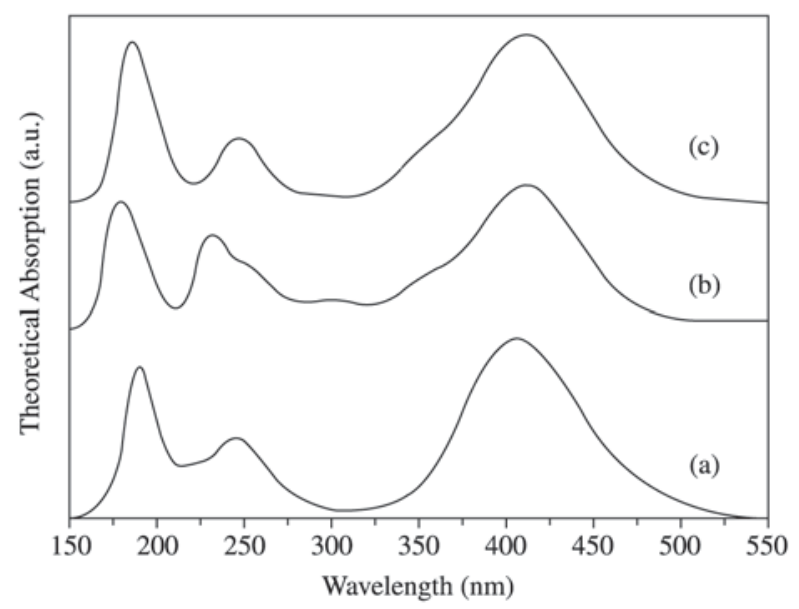

Figure 5. Theoretical absorption spectra. (a) $\mathrm{CV}^{+} / \mathrm{CV}^{+}$, (b) $\mathrm{MG}^{+} /$ $\mathrm{MG}^{+}$and (c) $\mathrm{CV}^{+} / \mathrm{MG}^{+}$dimers in parallel structure by INDO/S-CI (absorption spectra) using the semiempirical method calculated for optimized geometries.

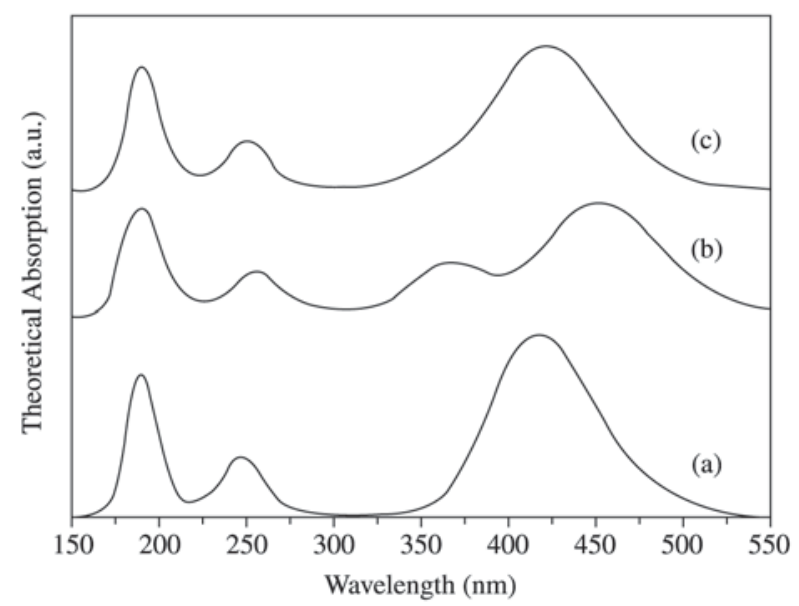

Figure 6. Theoretical absorption spectra . (a) $\mathrm{CV}^{+} / \mathrm{CV}^{+}$, (b) $\mathrm{MG}^{+} /$ $\mathrm{MG}^{+}$and (c) $\mathrm{CV}^{+} / \mathrm{MG}^{+}$dimers in anti-parallel structure by INDO/ $\mathrm{S}-\mathrm{CI}$ (absorption spectra) using the semiempirical method calculated for optimized geometries.
For the parallel $\mathrm{MG}+/ \mathrm{MG}+$ (Fig. 5b) interaction the most important absorption line corresponds to the $\mid \mathrm{H} \rightarrow \mathrm{L}>$ transition (coefficient -0.67 and o.s. $=0.12$ ), while the band at $\approx$ $232 \mathrm{~nm}$ has contributions from the $\mid \mathrm{H}-1 \rightarrow \mathrm{L}+2>$ transition (with coefficients of 0.48 ) and o.s. $=0.27$ and the first two bands are composed by only lower transitions.

For the parallel CV+/MG+ (Fig. 5c) interaction there are five main bands centered respectively in: (i) $453 \mathrm{~nm}$ region, composed by a $\mid \mathrm{H} \rightarrow \mathrm{L}>$ transition with o.s. $=0.15$, (ii) 422 $\mathrm{nm}$, with o.s. $=0.68(0.5 \mid \mathrm{H}-1 \rightarrow \mathrm{L}+1>$ and $-0.3 \mid \mathrm{H}-2 \rightarrow \mathrm{L}+1>$ transitions), (iii) $407 \mathrm{~nm}$, with o.s. $=1.93$ (the most important and corresponding to $0.3 \mid \mathrm{H} \rightarrow \mathrm{L}>$ and $-0.5 \mid \mathrm{H}-2 \rightarrow \mathrm{L}+1>$ transitions), (iv and v) $358 \mathrm{~nm}$ and 243 corresponding by a $\mid \mathrm{H}-3 \rightarrow \mathrm{L}>$ and an overlapping of $-0.33 \mid \mathrm{H}-1 \rightarrow \mathrm{L}+6>$ and $0.33 \mid \mathrm{H}-2 \rightarrow \mathrm{L}+3>$ with o.s. $=0.80$ and 0.22 , respectively.

Figure 6 represents the theoretical absorption spectra for anti-parallel dimer of (a) $\mathrm{CV}+/ \mathrm{CV}+$, (b) $\mathrm{MG}+/ \mathrm{MG}+$ and (c) $\mathrm{CV}+/ \mathrm{MG}+$ molecules interacting following the propose of Coon et $a l .{ }^{28}$. A different behavior is followed by antiparallel structures. There is a pronounced red shift for all species proportioned by strong charge interaction between the rings ${ }^{29}$. For $\mathrm{CV}+/ \mathrm{CV}+$ (Fig. 6a) the band at $\approx 430 \mathrm{~nm}$ has an o.s. equal to 0.68 and corresponds to a mixing of the transitions $\mid \mathrm{H} \rightarrow \mathrm{L}+1>$ and $|\mathrm{H}-1 \rightarrow \mathrm{L}\rangle$ (with coefficients of 0.35 and -0.59 , respectively). In Table 1 , note that the in the main transitions shows the importance of deeper and lower orbitals. For MG+/MG+ (Fig. 6b) appears a more pronounced red shift than the others two spectra and this behavior is according to the appearance of only lower orbitals. Similar analysis for $\mathrm{CV}+/ \mathrm{MG}+($ Fig. 6c) is done and it can see with Table 1.

\section{Conclusions}

The organic dyes crystal violet and malachite green were successfully incorporated within the aluminum polyphosphate gel inorganic matrix. The dyes are sensitive to changes in the chemical environment such as drying and exposure to acidic and basic vapors, which led optical responses (color changes) on the samples.

Theoretical investigation for the ground state and first excited states of MG and CV molecules, and examined the corresponding absorption spectra.

\section{Acknowledgments}

Support by the Brazilian agency PIBIC for JAPS (10060/2001) and Pernambuco state agency FACEPE for the fellowship for SBCS and financial support is gratefully acknowledged.

\section{References}

1. Sanchez, C.; Soler_illia, G.J.A.A.; Ribot, F.; Lalot, T.; Mayer, C.R.; Cabuil, V. Chem. Mater, n. 12, p. 3061, 2001. 
2. Lebeau, B.; Sanchez, C. Curr. Op. Solid State Mat. Sci, n. 4, p. 11, 1999.

3. Lima, E.C.O.; Galembeck, F. Colloid Surface A, n. 75, p. 65, 1993.

4. Galembeck, F.; Lima, E.C.O.; Beppu, M.M.; Sassaki, R.M.; Masson, N.C.; Monteiro, V.A.R.; Souza, E.F. "Fine Particles Science and Technology-: From Micro to Nanoparticles" (E. Pellizetti, Ed.), p. 267. Kluwer, Amsterdam, 1996.

5. Lima, E.C.O.; Galembeck, F.; Colloid, J. Interf. Sci., n. 166, p. 309, 1994.

6. Corbridge, D.E.C. Phosphorus, and Outline of its Chemistry, Biochemistry and Technology. Elsevier, Amsterdam, 1990.

7. Souza, E.F.; Bezerra, C.C.; Galembeck, F. Polymer, n. 38, p. 6285, 1997.

8. Amorim, M.S.; Galembeck, A.; Bazin, H.; Mathis, G.; Donegá, C.M.; Sá, G.F.; Junior, S.A. Mol. Cryst. Liq. Cryst., n. 374, p. 267, 2002.

9. Castro, E.G.; Zarbin, A.J.G.; Galembeck, A.; Del Nero, J. International Conference of Synthetic Metals 2002, Xangai, China, 2002.

10. Galembeck, A.; Silva, S.B.C.; Silva, J.A.P.; Del Nero, J. Opt. Mat., accepted, 2002.

11. Del Nero, J.; Silva, J.A.P.; Silva, S.B.C.; Galembeck, A. Synth. Met., in press.

12. Lovell, S.; Marquardt, B.J.; Kahr, B. J Chem. Soc. Perk. T. 2, p. 2241, 1999.

13. Lueck, H.B.; Rice, B.L.; McHale, J.L. Spectrochimica Acta, 48A, p. 819, 1992.
14. Raimundo Jr., I.M.; Narayanaswamy, R. Sensors Actuators, $B$ 74, p. 60, 2001.

15. Malins, C.; Butler, T.M.; MacCraith, B.D. Thin Solid Films, n. 368, p. 105, 2000.

16. Dewar, M.J.S.; Zoebisch, E.G.; Healy, E.F.; Stewart, J.P. J. Am. Chem. Soc., n. 107, p. 3902, 1985.

17. Stewart, J.J.P. MOPAC Program Version 6 (QCEP 455).

18. Head, J.D.; Zerner, M.C. Chem. Phys. Lett., n. 122, p. 264, 1985.

19. Head, J.D.; Zerner, M.C. Chem. Phys. Lett., n. 131, p. 359, 1986.

20. Ridley, J.; Zerner, M.C. Theoret. Chim. Acta, n. 42, p. 223, 1976.

21. Edwards, W.D.; Zerner, M.C. Theoret. Chim. Acta, n. 72, p. 347, 1987.

22. Del Nero, J.; Laks, B. Synth. Met., n. 101, p. 440, 1999.

23. Del Nero, J.; Laks, B. Synth. Met., n. 101, p. 379, 1999.

24. Doretto, R.L.; Del Nero, J.; Laks, B. Synth. Met., n. 101, p. 178, 1999.

25. Del Nero, J.; Melo, C.P. Synth. Met. n. 121, p. 1741, 2001.

26. Hoppe, R.; Alberti, G.; Costantino, U.; Dionigi, C.; Schulz-Ekloff, G.; Vivani, R. Langmuir, n. 13, p. 7252, 1997.

27. Del Nero, J.; Melo, C.P. Mol. Cryst.-Liq.Cryst., n. 374, p. 555, 2002.

28. Coon, S.R.; Zakharian, T.Y.; Littlefield, N.L.; Loheide, S.P.; Puchkova, E.J.; Freeney, R.M.; Pak, V.N. Langmuir, n. 16, p. $9690,2000$.

29. Lueck, H.B.; McHale, J.L.; Edwards, W.D. J. Am. Chem. Soc., n. 114, p. 2342, 1992. 